\title{
Asthma questionnaires
}

\section{Les questionnaires sur l'asthme}

I

$n$ the present issue of the Canadian Respiratory Journal, Ahmed et al (1) report the results of a study to validate The 30 Second Asthma Test ${ }^{\mathrm{TM}}$, a questionnaire derived from the 1995 Canadian Asthma Consensus Guidelines (2), and promoted by GlaxoSmithKline. The questionnaire assesses the presence or absence in the past week of daytime symptoms, night-time symptoms, rescue medication use, and limitation of physical activity and absenteeism in the previous three months. A dichotomous scoring system was used: if the patient's answers exceeded the threshold of 'good control' for two or more of the questions, control was judged to be bad, if not, control was good. These results were compared with data acquired at the same clinical visit including two specialists' rating of asthma control (on a five-point scale), decreases of forced expiratory volume in $1 \mathrm{~s}$ from the best value recorded previously, and changes in therapy resulting from the visit.

The 81 patients were adults and probably represented a 'real world' sample of specialty practice, including the fact that $34 \%$ of them were smokers.

Approximately 25\% of the patients were judged to have fair or poor control by the specialists, and not surprisingly, approximately the same number had decreases in forced expiratory volume in $1 \mathrm{~s}$ and intensification of therapy. Approximately $30 \%$ of the patients had 'bad control' as measured by the questionnaire, somewhat, but not much, more than the alleged gold standards. Only 5\% of the patients' answers to the questionnaire indicated good control when the physician thought otherwise. Overall, the sensitivity of the questionnaire was $81 \%$, and the specificity was $60 \%$; increasing the number of symptoms required for the designation of bad control increased specificity but decreased sensitivity. Ahmed et al (1) recommend using the sensitive cutoff of two positive answers.

I think that we can regard The 30 Second Asthma Test ${ }^{\mathrm{TM}}$ as validated at least insofar as an objective analysis of expert physicians' opinions is concerned. This is reassuring although not surprising, because symptom evaluation is presumably the cornerstone of the physician encounter. Asthma questionnaires are widely recognized as a valuable tool in asthma research and management, although most try to quantify severity as well as presence of symptoms. One of them, the Juniper Asthma Control Questionnaire (3), a product of the McMaster group, is regarded as a gold standard by many investigators for defining asthma control. Ahmed et al (1) publish a useful comparison of established questionnaires in their paper. The popularity of asthma questionnaires testifies to their ease of administration and simplicity of scoring, but also bears witness to the fact that symptom evaluations are the best correlates to the state of the patient. Recently, McCoy et al (4) had the opportunity to compare responses with symptom scores and ans le présent numéro du Canadian Respiratory Journal, Ahmed et coll. (1) présentent les résultats d'une étude pour valider le Test de 30 secondes sur l'asthme ${ }^{\mathrm{MC}}$, un questionnaire dérivé des Principes directeurs du consensus canadiens sur l'asthme de 1995 (2), dont GlaxoSmithKline fait la promotion. Le questionnaire permet d'évaluer, au cours de la semaine précédente, la présence ou l'absence de symptômes diurnes, de symptômes nocturnes, d'utilisation de médicaments de rattrapage et les limites d'activité physique et d'absentéisme au cours des trois mois précédents. On utilise un système de pointage dichotomique : si les patients dépassaient le seuil de «bonne maîtrise » dans au moins deux questions, l'asthme était bien maîtrisé. Autrement, il était mal maîtrisé. Ces résultats étaient comparés à des données acquises à la même consultation clinique grâce à l'évaluation de la maîtrise de l'asthme par deux spécialistes (sur une échelle de cinq points), aux diminutions du volume expiratoire maximal par seconde par rapport à la meilleure valeur enregistrée auparavant et aux modifications au traitement par suite de la consultation.

Les 81 patients étaient des adultes et représentaient probablement un échantillon « du monde réel » en pratique spécialisée, y compris le fait que $34 \%$ d'entre eux étaient fumeurs.

Les spécialistes ont évalué qu'environ $25 \%$ des patients maîtrisaient correctement ou mal leur asthme et, comme il fallait s'y attendre, environ le même nombre de patients présentaient une diminution du volume expiratoire maximal par seconde et avaient dû intensifier leur traitement. Environ $30 \%$ des patients « maîtrisaient mal » leur asthme d'après le questionnaire, soit un résultat quelque peu plus élevé que la prétendue norme de référence, mais pas tellement. Seulement $5 \%$ des réponses des patients faisaient état d'une «bonne maîtrise » tandis que le médecin pensait autrement. Dans l'ensemble, la sensibilité du questionnaire était de $81 \%$, et sa spécificité, de 60 \%. L'augmentation du nombre de symptômes nécessaires pour désigner une «mauvaise maîtrise » accroissait la spécificité, mais diminuait la sensibilité. Ahmed et coll. (1) recommandent d'utiliser le seuil sensible de deux réponses positives.

Je pense qu'on peut considérer le Test de 30 secondes sur l'asthme ${ }^{\mathrm{MC}}$ comme validé, du moins pour ce qui est d'une analyse objective des opinions de médecins experts. C'est rassurant, mais pas surprenant, car l'évaluation des symptômes constitue probablement la pierre angulaire de la rencontre avec le médecin. Les questionnaires sur l'asthme sont largement reconnus comme des outils précieux pour la recherche sur l'asthme et la prise en charge de cette maladie, bien que la plupart tentent de quantifier la gravité tout autant que la présence des symptômes. De nombreux chercheurs considèrent l'un d'eux, le questionnaire Juniper sur la maîtrise de l'asthme (3), un produit du groupe McMaster, comme une référence pour 
subsequent episodes of poor asthma control and found that in adults these scores were by far the best single predictors of future problems. Interestingly, in children younger than 10 years of age, they were much less accurate, probably indicating a communication problem. The symptom score used was a relatively simple one, dealing with four symptoms (cough, wheeze, dyspnea and nighttime awakenings) over the preceding two weeks.

The bottom line is that a systematic symptom inventory is likely the most valuable single tool in asthma assessment, both in the laboratory and in the clinic. While the questionnaire validated by Ahmed et al (1) is less sophisticated in quantitative terms than others, it is systematic and it does inquire about the important symptoms. Therefore, The 30 Second Asthma Test ${ }^{\mathrm{TM}}$ should have value if only as a checklist for the physician who sees asthmatics.

Nick R Anthonisen MD Editor-in-Chief, Canadian Respiratory Journal

\section{REFERENCES}

1. Ahmed S, Ernst P, Tamblyn R, Colman N. Validation of The 30 Second Asthma Test ${ }^{\mathrm{TM}}$ as a measure of asthma control. Can Respir J 2007;2:105-9.

2. Ernst P, FitzGerald JM, Spier S. Canadian Asthma Consensus Conference: Summary of Recommendations. Can Respir J 1996;3:89-100.

3. Juniper EF, O'Byrne PM, Guyatt GH, Ferrie PJ, King DR. Development and validation of a questionnaire to measure asthma control. Eur Respir J 1999;14:902-7.

4. McCoy K, Shade DM, Irvin CG, et al, for the American Lung Association Asthma Clinical Research Centers. Predicting episodes of poor asthma control in treated patients with asthma. J Allergy Clin Immunol 2006;118:1226-33. définir la maîtrise de l'asthme. Dans leur article, Ahmed et coll. (1) effectuent une comparaison utile des questionnaires bien établis. La popularité des questionnaires sur l'asthme témoigne de leur facilité d'administration et de la simplicité du pointage, mais également du fait que les évaluations des symptômes sont les meilleurs corrélats de l'état du patient. Récemment, McCoy et coll. (4) ont pu comparer les réponses avec le pointage des symptômes et les épisodes subséquents de mauvaise maîtrise de l'asthme et ont découvert que, chez les adultes, ce pointage était de loin le meilleur prédicteur unique de futurs problèmes. Il est intéressant de constater que, chez les enfants de moins de dix ans, les questionnaires étaient beaucoup moins précis, ce qui indique probablement un problème de communication. Le pointage utilisé était relativement simple, puisqu'il portait sur quatre symptômes (toux, respiration sifflante, dyspnée et réveils nocturnes) au cours des deux semaines précédentes.

Au bout du compte, l'inventaire systématique des symptômes constitue, selon toute probabilité, le meilleur outil unique pour évaluer l'asthme, tant en laboratoire qu'en clinique. Le questionnaire validé par Ahmed et coll. (1) est moins subtil que d'autres au point de vue quantitatif, mais il est systématique et porte sur les symptômes importants. Par conséquent, le Test de 30 secondes sur l'asthme $\mathrm{MC}^{\mathrm{MC}}$ est utile, si ce n'est qu'à titre de liste de vérification pour le médecin qui soigne des asthmatiques.

Nick R. Anthonisen MD Rédacteur en chef, Canadian Respiratory Journal

\section{RÉFÉRENCES}

1. Ahmed S, Ernst P, Tamblyn R, Colman N. Validation of The 30 Second Asthma Test ${ }^{\mathrm{TM}}$ as a measure of asthma control. Can Respir J 2007;2:105-9.

2. Ernst P, FitzGerald JM, Spier S. Canadian Asthma Consensus Conference: Summary of Recommendations. Can Respir J 1996;3:89-100.

3. Juniper EF, O'Byrne PM, Guyatt GH, Ferrie PJ, King DR. Development and validation of a questionnaire to measure asthma control. Eur Respir J 1999;14:902-7.

4. McCoy K, Shade DM, Irvin CG, et al, for the American Lung Association Asthma Clinical Research Centers. Predicting episodes of poor asthma control in treated patients with asthma. J Allergy Clin Immunol 2006;118:1226-33. 


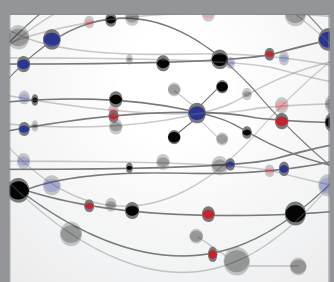

The Scientific World Journal
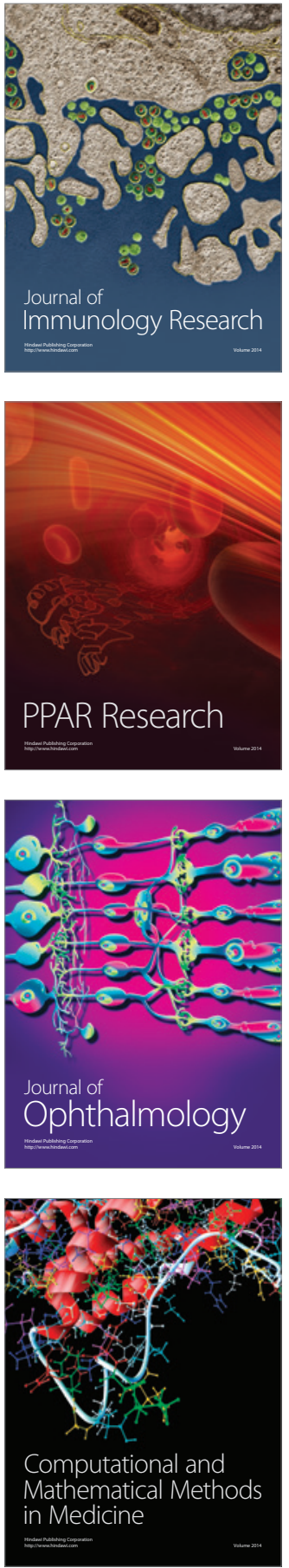

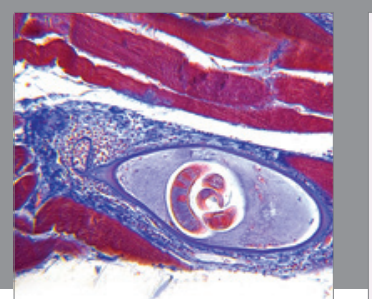

Gastroenterology Research and Practice

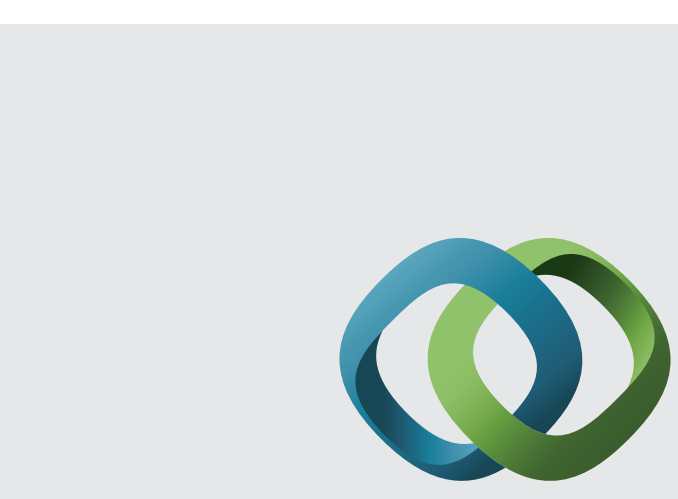

\section{Hindawi}

Submit your manuscripts at

http://www.hindawi.com
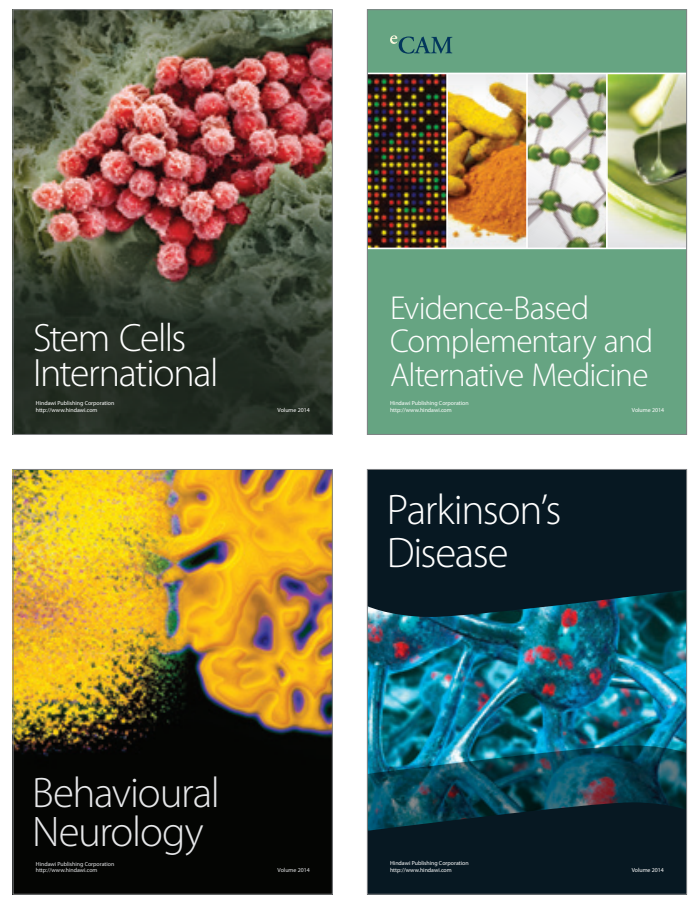
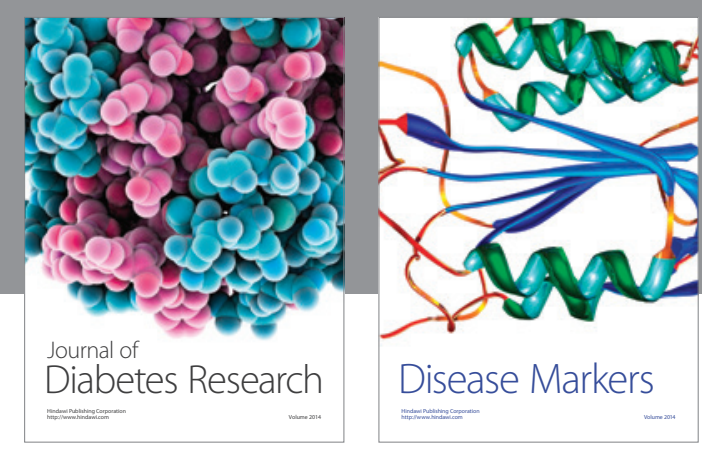

Disease Markers
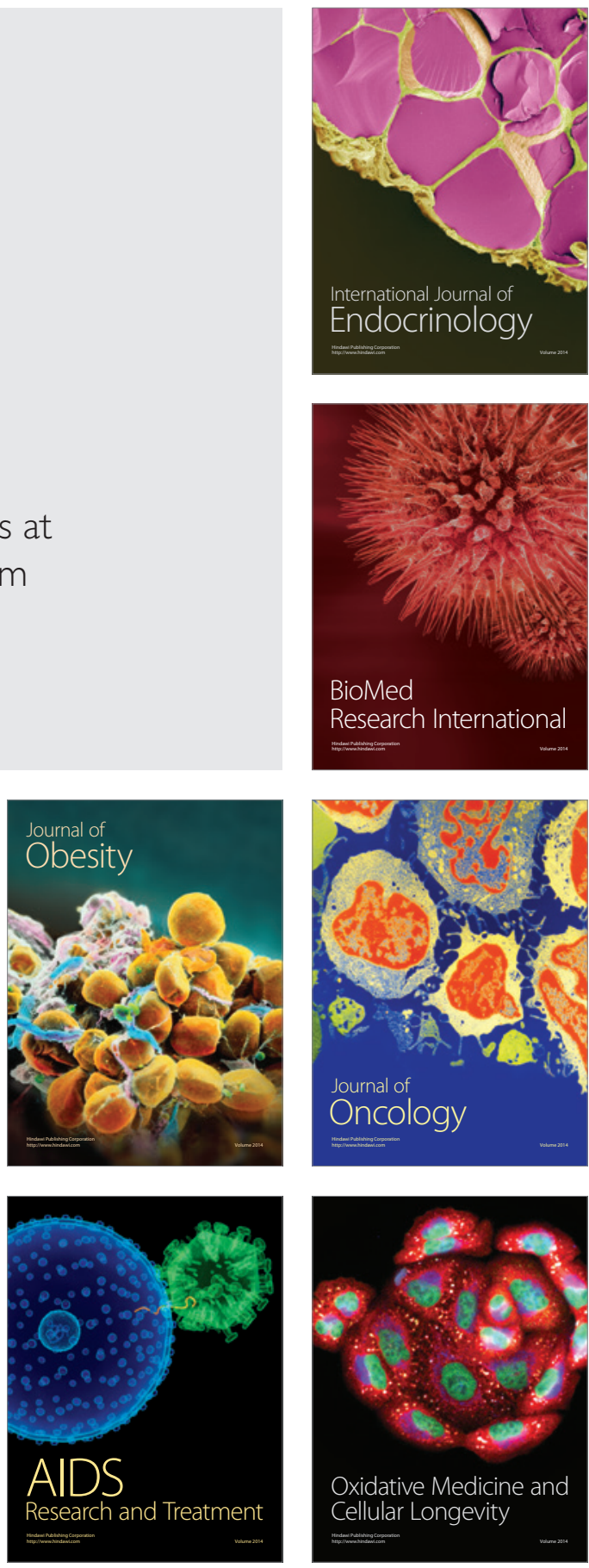